\title{
The Murky Distinction Between Curiosity and Interest: State of the Art and Future Prospects
}

\author{
Reinhard Pekrun ${ }^{1,2}$ \\ Published online: 5 December 2019 \\ (C) The Author(s) 2019
}

\begin{abstract}
Curiosity and interest are at the core of human inquiry. However, controversies remain about how best to conceptualize these constructs. I propose to derive definitions by attending to the common core of typical usages of the two terms. Using this approach, curiosity can be defined as a psychological state that includes three components: recognition of an information gap, anticipation that it may be possible to close it, and an intrinsically motivated desire to do so. Interest can be more broadly defined as intrinsically motivated engagement with any specific object, content, or activity. The two definitions imply that curiosity is a special case of interest. Furthermore, I propose to use the state-trait distinction to distinguish between momentary and enduring forms of both curiosity and interest, which makes it possible to treat state versus trait curiosity and interest in conceptually parallel ways. To make further progress in understanding the two constructs, research is needed that investigates their affective dynamics and their generalizability across age-related and socio-cultural contexts.
\end{abstract}

Keywords Curiosity $\cdot$ Interest $\cdot$ Jingle-jangle fallacy $\cdot$ Information gap $\cdot$ Control-value theory

Curiosity and interest are central to the generation of knowledge. As such, the two constructs are critically important across scientific disciplines. Furthermore, they are important not only from a research perspective but for practice in education and science as well. Given their relevance, it is important to have a clear, common understanding of how best to conceptualize these two constructs. Yet multiple controversies remain on how to define them. This situation hinders scientific communication; is reflected in measurement instruments that are not equivalent across programs of research, which impedes building consistent knowledge; and, by implication, makes it difficult to derive recommendations for practice. As aptly summarized by

Reinhard Pekrun

pekrun@1mu.de

1 Department of Psychology, University of Munich, 80802 Munich, Germany

2 Institute for Positive Psychology and Education, Australian Catholic University, Sydney, Australia 
Shin and Kim (2019 in this issue), the field is built "on a foggy conceptual foundation ... [which] significantly limits educational applicability."

What can we do to make progress? Each of the contributions in this special issue provides an intriguing, multifaceted view on how this could be achieved. Taken together, these articles provide a comprehensive picture of the state of the art in the field. Using these contributions as a starting point, I propose that we employ three interrelated strategies for future inquiry. First, it seems necessary to reach more of a consensus on issues of terminology. Second, to support this aim, we need more in-depth research on the affective dynamics of curiosity and interest. Third, I recommend better contextualizing the two constructs in age-related and socio-cultural contexts that may (or may not) shape their components, processes, and functions.

\section{Terminology: How to Define Curiosity and Interest?}

The contributions in this special issue provide rich conceptualizations of the two constructs. However, there is substantial divergence among the proposed concepts, especially for state (i.e., momentary) curiosity and interest. The two states are seen as almost the same by Ainley who notes that they are intertwined, have "very similar observable features," and "are often indistinguishable." In contrast, while acknowledging that the two states can overlap, Hidi and Renninger as well as Peterson and Cohen emphasize that curiosity and interest are nevertheless distinguishable. Hidi and Renninger argue that they can differ in terms of "affective markers," duration, and triggers, with curiosity being primarily linked to cognitive uncertainty and interest to specific contents. From Peterson and Cohen's perspective, awareness of a knowledge gap, an urge to close the gap, and a sense that this can be achieved are core components of curiosity, whereas interest is conceptualized as a more general desire to increase one's knowledge. The extreme opposite from Ainley's position is represented in Shin and Kim's contribution suggesting that curiosity and interest are completely disjunct states. In their view, curiosity is a state of perceived deprivation generated by uncertainty and accompanied by negative affect, whereas interest involves attention that is captured by the hedonic experience of interacting with certain stimuli, rendering interest a positive affective state comprising enjoyment and pleasure. Finally, given these diverging views, Murayama et al. propose that it may be better to simply refrain from using the concepts at this stage of research.

\section{Strategies to Define Concepts}

How could we reduce the gaps between different research traditions as represented by these diverging conceptualizations? Five major options are listed below. Importantly, whereas the first and the subsequent four options are mutually exclusive, the latter four options can be combined.

Option 1: Dropping Terms Given conceptual confusion about a term, it is possible to drop it from scientific discourse. One variant is to stop labeling the phenomenon denoted by the term altogether. However, this solution is not viable if one wants to communicate about the phenomenon, which is necessary to make progress in investigating it. Another variant is to replace existing terms with new terms, as done by Murayama et al. (2019) when dropping the two terms. Instead, they talk about the "rewarding experience" or "feeling of reward" that is associated with learning and drives "autonomous knowledge acquisition." However, as long as 
a new term is not defined more clearly than the old one, this variant does not resolve the problem either. Terms like feeling of reward may be fuzzy concepts as well - what are the components of this experience, and what exactly is a "reward"?

Option 2: Definition by Common Core Scientific terms serve communication. To enable smooth communication and avoid misunderstandings, it is often best to simply use the common core of typical usages of a term to define it (Pekrun 1988). The added value of this approach is that resulting definitions are generally less complex and clearer than existing ones. For example, "personality" has been defined in a myriad of ways, with many proposed definitions including a broad range of definitional components that diverge across authors (see, e.g., Feist et al. 2018). When using the common core principle, it is easy to derive a consensual, less complex, and more precise definition. According to this definition, "personality" denotes the set of human characteristics that (a) can vary between persons and (b) are relatively stable over time (Guilford 1959; Pekrun 1988). No more than these two features are needed to conceptualize personality in a precise and efficient way. Similarly, it should be possible to derive parsimonious definitions of curiosity and interest by using the common core principle (see below). Importantly, this principle can be used to synthesize existing definitions regardless of how they are constructed (e.g., based on components, causes, prototypes, or lists of category members as described in options 3-5).

Option 3: Definition by Components and Causes If phenomena conceptually comprise more than one single, unitary element, it can be useful to define them by denoting these elements and depicting their status within the definition (e.g., some elements may be required to define membership in a category, whereas others are optional or required only for prototypical cases). In addition, categories can be defined based on common causes of phenomena, such as diseases being defined not only by their symptoms but also their etiology. Most curiosity and interest definitions proposed in this special issue use a component definition approach. However, to make such definitions effective, it is important to assemble components in nonarbitrary ways. As suggested early on, for example, by Allport (1937), it makes sense to define psychological concepts based on structural and functional covariation of elements. This also makes it possible to empirically scrutinize the usefulness of adding specific components, thus making definitions in part amenable to empirical investigation. For example, if we consider the perception of an information gap as a necessary constituent of curiosity and an unpleasant experience of deprivation as a possible additional component that could be included in the definition, then it is possible to examine the usefulness of this addition by examining co-occurrence of information gaps and a sense of deprivation. In a similar way, the usefulness of adding positive feelings to the definition of interest can be investigated empirically.

Option 4: Definition by Prototype It is often useful to acknowledge that some members of a category are conceptually more representative of the category than others and to use these exemplars to describe the category. For example, joy, anger, and anxiety are affective states that are at the core of the category "emotion." Other states may be less representative although still regarded as members of the family. Curiosity is a case in point. Given strong cognitive and motivational connotations, curiosity can be classified as cognition or motivation, but given its multicomponent nature including affective and physiological processes as well, it can also be considered an emotion (Brun et al. 2008; Pekrun and Stephens 2012). Similarly, different 
variants of curiosity may be more or less representative of the category of curiosity, and different variants of interest of the category of interest.

Option 5: Definition by Listing Variants Related to the prototype approach, it is possible to define categories by denoting specimens that make up the category. One version of this strategy is to create summative lists of members of the category. However, this may be less satisfying than defining a conceptual core of different members and then defining variants that differ in terms of additional components. The various types of trait curiosity and individual interest that are distinguished in the literature are examples for this strategy.

\section{Implications: How Should We Define Curiosity and Interest?}

Which of the above options should we use? As argued earlier, the first option (dropping the terms) is likely not the best way to promote scientific communication. Instead, I propose to use the common core approach. This can be done both for momentary states of curiosity and interest that occur in a specific situation at a specific point in time and for trait curiosity and interest that represent enduring dispositions to experience these states. For interest, the terms "situational" and "individual" interest are used in the current literature to denote the state-trait distinction (see Hidi \& Renninger 2019, and Renninger and Hidi 2016). For three reasons, it may be preferable to use the terms state and trait. First, not only states but traits as well can be situationally specific (e.g., a student's disposition to habitually be curious in mathematics is a situation-specific trait). The term "state" may be better suited to denote temporal rather than situation-related specificity. Second, the terms state and trait are aligned with the general psychological literature. Third, they make it possible to discuss curiosity and interest in conceptually parallel ways.

In proposing the term "trait" to denote dispositions to repeatedly experience curiosity or interest, I use the term as it is employed in research on personality. In this field, "trait" is a descriptive category that denotes habitual patterns of thought, affect, and action (Deary 2009; Matthews 2018), such as habitual emotions in trait anxiety or trait anger. Used this way, the term does not imply that traits involve genetic predispositions, would need to be generalized across different types of situations, or cannot change. Traits can be but need not be dependent on genotype (the heritability of personality traits is moderate at best; Sanchez-Roige et al. 2018), can be situation-specific as noted, and typically change across the lifespan. As such, habitual curiosity as well as individual interest fit into the broad category of traits.

State Curiosity and Interest Scrutinizing different proposals to define state curiosity, one common element seems to be that curiosity is a psychological state related to an information gap. Variants of the term that do not explicitly refer to information gaps can at least be reconstructed to represent such gaps, such as perceptual curiosity which refers to a gap between current and desired perceptual knowledge about perceived objects, or diversive curiosity which refers to a gap between current knowledge and the as yet unknown, expanded knowledge that could be gained by unspecific exploration (see Murayama et al., 2019, for a similar view). Even cases where the immediate situational appraisal does not refer to an information gap but to other types of cognitive incongruence can be considered as representing information gaps. For example, when reading contradictory texts, the related immediate 
appraisal is cognitive incongruence, but at the meta-level, there is a gap between incongruence and the desired state of congruence.

However, referring to information gaps may not be sufficient to define state curiosity. First, information gaps may alternatively cause surprise, confusion, or frustration. As aptly argued by Peterson and Cohen (2019), what needs to be added is a sense of control that it will be possible to close the gap. This is also represented in Loewenstein's (1994; Markey and Loewenstein 2014) proposition that a medium information gap triggers curiosity, whereas gaps that are too wide do not, and in Pekrun's (2019) control-value approach to curiosity. Second, the common core of usages is that curiosity involves the desire to see closure of an information gap for its own sake, regardless of any instrumental functions of closing the gap. In other words, curiosity relates to closing an information gap because closure has intrinsic value. An information gap that one wants to close for extrinsic reasons (e.g., in order to pass an exam) does not need to trigger curiosity. In sum, using the common core principle, curiosity can be defined as a psychological state that includes recognition of an information gap, combined with a sense that closing the gap is possible and intrinsically valuable.

Beyond the core components of knowledge gap, sense of control, and intrinsic value, additional components may be amended to define variants of curiosity. However, at this stage of research, it seems there is insufficient evidence to consider additional components as necessary constituents of curiosity. For example, while it is plausible to assume that information gaps can be associated with a negatively valenced state of deprivation, it remains unclear how often and under what conditions this is the case, as argued by Murayama et al. (2019).

For state interest (i.e., situational interest), the common core of definitions in psychology and education seems to be engagement with an activity, object, or content because such engagement is intrinsically valuable, similar to the desire to close an information gap regardless of extrinsic rewards when feeling curious. As with curiosity, agreement on additional components would require more evidence. Classical definitions of interest take this into account by regarding additional components as possible, but not necessary, ingredients. For example, Hidi and Renninger (2019) note that situational interest can be associated with either positive or negative feelings, which implies that positive emotion is not a necessary component. Positive emotion may be useful to delineate prototypical cases of state interest, but there are cases in which interest involves negative emotions or blends of positive and negative emotions (e.g., students' disgust when being fascinated by dissecting frogs; Holstermann et al. 2012).

Trait Curiosity and Interest Trait curiosity is commonly defined as individuals' disposition to frequently experience curiosity across a broad range of situations. In contrast, trait interest (i.e., individual interest) is defined as an individual's disposition to reengage with specific activities, objects, or contents. As such, at the trait level, there is an asymmetry between the two constructs in terms of situational specificity. It remains open to question whether this asymmetry could (or should) be reduced or even completely eliminated at the conceptual level. As outlined in the contribution by Peterson and Cohen, trait curiosity could well be conceptualized in domain-specific terms (domain-specific trait curiosity). This possibility may have been overlooked by trait curiosity researchers who often use dimensional personality theories as a conceptual basis. These theories (e.g., the Big Five factor model) tend to neglect the possibility that traits can be domain-specific (see also Matthews 2018). 
In a complementary way, it might make sense to conceptualize trait interest as a domaingeneral disposition, in addition to single specific interests. Based on the common core definition outlined above, such a construct would represent an individual's disposition to frequently experience interest across a broad range of situations, that is, a general propensity to engage with activities, objects, or contents (domain-general trait interest). Such a general trait would be closely related to a person's habitual activity level, vitality, and openness, dimensions that have been considered in research on temperament and personality (e.g., Buss and Plomin 1984).

Relations Between Curiosity and Interest: the Same or Different? From common core definitions, it follows that curiosity and interest are neither the same nor completely disjunct. Rather, it follows that they are overlapping. More specifically, the above definitions imply that curiosity is a special case of interest: Curiosity is a state of engaging with a specific object, namely an information gap, and motivates a specific activity related to this object, namely closing the information gap, regardless of any extrinsic rewards. From this perspective, the overall relation between the two constructs represents a part-whole relationship rather than a relationship between mutually exclusive concepts. States of curiosity represent a subset of states of interest. If interest relates to closing an information gap, combined with a sense that this is possible and intrinsically rewarding, then curiosity and interest are the same. Given that learning can be defined as closing (or reducing) information gaps, during learning interest is conceptually identical with curiosity if there is a sense of agency. Similarly, from the perspective of common core definitions, trait curiosity and trait interest can be conceptualized as overlapping constructs.

The overlap between curiosity and interest as based on common core definitions has important implications for measurement, intervention, and practice. Especially for curiosity and interest during learning, given the overlap, it may be justified to construct molar measures that assess the two constructs combined (e.g., Pekrun et al. 2017). Similarly, it should be possible to design classroom interventions and reform educational practices in ways that simultaneously foster both curiosity and interest.

\section{Investigating the Affective Dynamics of Curiosity and Interest}

Research on curiosity and interest has begun to explore the affective processes that generate and characterize the two states, which is well reflected in all five contributions to this special issue. Curiosity is described as a desire to know, and more specifically as a desire to reduce information gaps and cognitive uncertainty, in all five articles. Interest is denoted as an urge to "gravitate towards certain stimuli" (Shin \& Kim 2019) and to engage with specific activities or contents. Furthermore, all five contributions acknowledge the primary role that emotions play in this process, such as surprise that can contribute to the awareness of information gaps; negative affect and feelings of discomfort when faced with such gaps; anticipatory positive emotions when expecting to close a gap; satisfaction, enjoyment, delight, and excitement, but possibly also negative emotions once the gap has been closed; and both positive and negative feelings associated with states of interest.

In-depth research investigating the emotions and motivational processes involved in curiosity and interest is needed to better understand them and to design interventions and derive 
recommendations for practice. Furthermore, the findings from such research could also help clarify how to best conceptualize the two constructs. For example, as noted earlier, empirical investigation could help determine under what circumstances curiosity is characterized by negative or positive affect. As argued by Murayama et al. (2019), it is possible that both options play a role, with negative affect created by the perceived discrepancy between current and desired knowledge, and positive feelings by the anticipation of being able to reduce the discrepancy. Alternatively, it seems possible that working on solving a cognitive discrepancy is enjoyable in itself, regardless of any anticipation of resolving it. Furthermore, it is possible that negative rather than positive anticipatory emotions could be generated when feeling doubtful if the discrepancy can be reduced. Similarly, as argued by Hidi and Renninger (2019), triggered interest can involve not only positive emotions but also negative feelings. Exploring these different possibilities in more depth requires advances in both theory and methodology.

In terms of theory, substantial progress has been made through microgenetic process models of curiosity, such as the intriguing models by Murayama et al. and Shin and Kim summarized in their contributions to this issue and the macrogenetic four-phase model of interest development proposed by Hidi and Renninger (2006). These models could be further refined and expanded by using principles of general theories of both emotion and motivation. For example, as proposed in the contribution by Peterson and Cohen, principles of the controlvalue theory (CVT; Pekrun 2006, 2018) could be used to explain the emotions created by information gaps. Using terms from CVT, both activity emotions and outcome emotions could be generated when working towards closing an information gap. Activity emotions can comprise enjoyment of working on closing the gap (e.g., when being in a state of flow during problem solving), as well as anger or frustration when faced with obstacles and impasses. Outcome emotions related to prospects of closing the gap can include enjoyable anticipation of closing the gap, or at least hope that this may be possible, but also anxiety or hopelessness in case this seems not possible. The resulting motivation to work on closing the information gap can be driven both by activity emotions and by prospective outcome emotions. Finally, once the gap has been closed (or not), retrospective outcome emotions, such as pride about success or shame about failure, can occur and drive the affective dynamics in the next cycle of acquiring further knowledge.

In many situations, it is subjectively uncertain what the outcome of a quest for knowledge will be. Motivation theory can help explain the affective processes that follow. For example, different curiosity states could be conceptualized using the distinction between approach and avoidance motivation. Curiosity coupled with an approach tendency would imply a focus on attaining resolution, with accompanying positive prospective emotions. Curiosity coupled with avoidance motivation would focus on wanting to avoid not being able to reduce discomfort triggered by the gap, with accompanying negative emotions such as fear and anxiety. Similarly, interest in generally expanding one's knowledge in a given content domain could be associated with positive or negative prospective emotions depending on the kind of motivational orientation involved.

In terms of methodology, three important ways to make progress may be the following. First, most studies have relied on self-report measures. While self-report is indispensable to gain a more nuanced picture of thoughts and feelings, it is limited to conscious experience and subject to recall biases and response sets like social desirability. By implication, self-report needs to be complemented with other channels of assessment such as behavioral observation, implicit measures, physiological analysis, and neuroimaging (see Hidi \& Renninger 2019; Murayama et al. 2019; and Shin \& Kim 2019). This is especially important when the goal is to 
assess the affective dynamics of curiosity and interest, which cannot be captured even by momentary self-report (e.g., experience sampling methodology) given that self-report inevitably lags behind and does not provide a moment-to-moment temporal resolution. Second, much of the existing research used correlational, cross-sectional designs, especially in research on interest. Experimental and controlled longitudinal research is needed to examine processes and cause-effect relations.

Finally, most existing studies, both experimental and correlational, have employed between-person analytic designs. Between-person research is suited to examine individual differences, but not suited to investigate the within-person psychological processes that characterize affective states such as curiosity and interest. To this end, within-person research is needed, best combined with a between-person approach in terms of analyzing both individual trajectories and variation of these trajectories across persons (see Murayama et al. 2017; Vogl, Pekrun, Murayama, \& Loderer 2019).

\section{Generalizability Across Age Groups and Socio-cultural Contexts}

The concepts used in research on curiosity and interest are typically based on investigations with student samples from Western countries. As such, assumptions on component structures and functional relations of the two constructs may also have been derived relative to Western student populations. This begs the question if it is adequate to use the concepts in the same way for other age groups and for populations around the world (see also Ainley 2019). In fact, for participants' subjective understanding of the constructs, there is evidence that there may be variation. For example, Frenzel et al. (2012) have shown that subjective conceptions of interest are primarily based on affective components in early adolescence, but shift to an emphasis on cognitive components during subsequent years. Does such variation in subjective concepts imply that different constructs would need to be conceptualized for different cultures and age groups?

A possible answer is that principles of relative universality, which I have proposed for achievement emotions (Pekrun 2006, 2018), apply for curiosity and interest as well. According to these principles, the objects, contents, frequencies, and process parameters (intensity, decay rates, etc.) of curiosity and interest can vary widely between individuals, genders, academic domains, age groups, and cultures. However, their component structures and relations with origins and outcomes should be equivalent across persons and contexts. It is the latter proposition that is most relevant for the definition of the two concepts: To the extent that components are invariant, it is possible to use definitions that are based on components in the same way across populations, content domains, and socio-cultural contexts. If components differ, it would be necessary to use different concepts to gauge curiosity and interest in different populations or under different circumstances.

While definitions of constructs are a matter of theory and communicative agreement in the first place, it is this aspect of definitions that is amenable to empirical scrutiny, as noted earlier. As such, examining generalizability may be an important avenue for future research on the two constructs. Research with different age groups and cross-cultural studies may be especially important for doing this. Several methodological paradigms can be used to this end, including qualitative work, quantitative analysis based on large-scale student assessments that comprised measures of curiosity or interest (e.g., the assessments of the OECD Programme for International Student Assessment [PISA] that included various measures of student interest across 
cycles; see, e.g., OECD 2017), or even cross-cultural studies of the linguistic profiles of related terms (Fontaine et al. 2013).

\section{Conclusion}

Definitions of psychological concepts cannot be "discovered" by empirical investigation. Rather, they need to be constructed by defining nomological networks that denote their components and their relations with origins and outcomes. By implication, given that theories can be construed in multiple ways, definitions are subject to theoretical preferences. If these preferences vary widely between authors, as is the case with curiosity and interest, then scientific communication and knowledge-building are jeopardized. To solve this problem, I propose we use the common core of current usages of the two terms as a starting point. Employing this principle, curiosity can be defined as a psychological state that is triggered by an information gap, combined with a sense that closing the gap is possible and desirable regardless of extrinsic rewards. Similarly, interest can be defined as engagement with a specific activity, object, or content regardless of extrinsic rewards. These common core concepts imply that curiosity is a special case of interest. However, to make further progress in understanding the two constructs, theoretical and methodological advances are needed, and to examine their generalizability, systematic research across age-related and socio-cultural contexts is required.

Acknowledgments I thank Kristina Loderer, Kathleen Quinlan, Elisabeth Vogl, and the editors of this special issue for helpful comments on an earlier version of this article.

Open Access This article is distributed under the terms of the Creative Commons Attribution 4.0 International License (http://creativecommons.org/licenses/by/4.0/), which permits unrestricted use, distribution, and reproduction in any medium, provided you give appropriate credit to the original author(s) and the source, provide a link to the Creative Commons license, and indicate if changes were made.

\section{References}

Ainley, M. (2019). Curiosity and interest: Emergence and divergence. Educational Psychology Review, 31. https://doi.org/10.1007/s10648-019-09495-z.

Allport, G. W. (1937). Personality: A psychological interpretation. New York: Holt, Rinehart, \& Winston.

Brun, G., Doğuoğlu, U., \& Kuenzle, D. (Eds.). (2008). Epistemology and emotions. Aldershot: Ashgate.

Buss, A., \& Plomin, R. (1984). Temperament: Early developing personality traits. Hillsdale: Erlbaum.

Deary, I. J. (2009). The trait approach to personality. In P. J. Corr \& G. Matthews (Eds.), The Cambridge handbook of personality psychology (pp. 89-109). New York: Cambridge University Press. https://oi. org/10.1017/CBO9780511596544.009.

Feist, J., Feist, G., \& Roberts, T.-A. (2018). Theories of personality (9th ed.). New York: McGraw-Hill.

Fontaine, J. J. R., Scherer, K. R., \& Soriano, C. (Eds.). (2013). Components of emotional meaning: A sourcebook. Oxford: Oxford University Press.

Frenzel, A. C., Pekrun, R., Dicke, A. L., \& Goetz, T. (2012). Beyond quantitative decline: Conceptual shifts in adolescents' development of interest in mathematics. Developmental Psychology, 48, 1069-1082. https://doi.org/10.1037/a0026895.

Guilford, J. P. (1959). Personality. New York: McGraw-Hill.

Hidi, S. E., \& Renninger, K. A. (2006). The four-phase model of interest development. Educational Psychologist, 41, 111-127. https://doi.org/10.1207/s15326985ep4102_4.

Hidi, S. E., \& Renninger, K. A. (2019). Interest development and its relation to curiosity: Needed neuroscientific research. Educational Psychology Review, 31. https://doi.org/10.1007/s10648-019-09491-3 
Holstermann, N., Roick, T., \& Bögeholz, S. (2012). The specific relationship between disgust and interest: Relevance during biology class dissections and gender differences. Learning and Instruction, 22, $185-192$. https://doi.org/10.1016/j.learninstruc.2011.10.005.

Loewenstein, G. (1994). The psychology of curiosity: A review and reinterpretation. Psychological Bulletin, 116, 75-98.

Markey, A., \& Loewenstein, G. (2014). Curiosity. In R. Pekrun \& L. Linnenbrink-Garcia (Eds.), International handbook of emotions in education (pp. 246-264). New York: Taylor \& Francis.

Matthews, J. G. (2018). Cognitive-adaptive trait theory: A shift in perspective on personality. Journal of Personality, 86, 69-82. https://doi.org/10.1111/jopy.12319.

Murayama, K., Goetz, T., Malmberg, L.-E., Pekrun, R., Tanaka, A., \& Martin, A. J. (2017). Within-person analysis in educational psychology: Importance and illustrations. In D. W. Putwain \& K. Smart (Eds.), British Journal of Educational Psychology Monograph Series II: Psychological Aspects of EducationCurrent Trends: The Role of Competence Beliefs in Teaching and Learning (pp. 71-87). Oxford: Wiley.

Murayama, K., FitzGibbon, L., \& Sakaki, M. (2019). Process account of curiosity and interest: A rewardlearning perspective. Educational Psychology Review, 31. https://doi.org/10.1007/s10648-019-09499-9.

OECD (Organisation for Economic Cooperation and Development). (2017). PISA 2015 results (Volume 3): Students' well-being. Paris: OECD Publishing.

Pekrun, R. (1988). Emotion, Motivation und Persönlichkeit [Emotion, motivation and personality]. München/ Weinheim: Psychologie Verlags Union.

Pekrun, R. (2006). The control-value theory of achievement emotions: Assumptions, corollaries, and implications for educational research and practice. Educational Psychology Review, 18, 315-341. https://oi. org/10.1007/s10648-006-9029-9.

Pekrun, R. (2018). Control-value theory: A social-cognitive approach to achievement emotions. In G. A. D. Liem \& D. M. McInerney (Eds.), Big theories revisited 2: A volume of research on sociocultural influences on motivation and learning (pp. 162-190). Charlotte: Information Age Publishing.

Pekrun, R. (2019). Self-appraisals and emotions: A control-value approach. Chapter prepared for T. Dicke, F. Guay, H. W. Marsh, R. G. Craven, \& D. M. McInerney (Eds). (forthcoming). Self - a multidisciplinary concept. Charlotte, NC: Information Age Publishing.

Pekrun, R., \& Stephens, E. J. (2012). Academic emotions. In K. R. Harris, S. Graham, T. Urdan, J. M. Royer, \& M. Zeidner (Eds.), APA educational psychology handbook (Vol. 2, pp. 3-31). Washington, DC: American Psychological Association.

Pekrun, R., Vogl, E., Muis, K. R., \& Sinatra, G. M. (2017). Measuring emotions during epistemic activities: The Epistemically-Related Emotion Scales. Cognition and Emotion, 31, 1268-1276. https://doi.org/10.1080 /02699931.2016.1204989.

Peterson, E. G., \& Cohen, J. (2019). A case for domain-specific curiosity in mathematics. Educational Psychology Review, 31, . https://doi.org/10.1007/s10648-019-09501-4.

Renninger, K. A., \& Hidi, S. (2016). The power of interest for motivation and engagement. New York: Taylor \& Francis / Routledge.

Sanchez-Roige, S., Gray, J. C., MacKillop, J., Chen, C. H., \& Palmer, A. A. (2018). The genetics of human personality. Genes, Brain and Behavior, 17(3), e12439. https://doi.org/10.1111/gbb.12439.

Shin, D. D., \& Kim, S. I. (2019). Homo curious: Curious or interested? Educational Psychology Review, 31. https://doi.org/10.1007/s10648-019-09497-x.

Vogl, E., Pekrun, R., Murayama, K., \& Loderer, K. (2019). Surprised - curious - confused: Epistemic emotions and knowledge exploration. Emotion. Advance online publication. https://doi.org/10.1037/emo0000578

Publisher's Note Springer Nature remains neutral with regard to jurisdictional claims in published maps and institutional affiliations. 